OESOPHAGEAL DISEASE

\title{
Biopsy surveillance is still necessary in patients with Barrett's oesophagus despite new endoscopic imaging techniques
}

\author{
K Egger, M Werner, A Meining, R Ott, H-D Allescher, H Höfler, M Classen, T Rösch
}

See end of article for authors' affiliations

\section{Correspondence to:} Dr T Rösch, Department of Internal Medicine II, Technical University of Munich, Klinikum rechts der Isar, Ismaningerstr 22, D-81675 München, Germany;

Thomas.Roesch@ Irz.tu-muenchen.de

Accepted for publication 24 June 2002

\begin{abstract}
Background and aims: Endoscopic surveillance including stepwise four quadrant biopsies $(4 Q B)$ is still regarded as the standard approach in patients with Barrett's oesophagus (BO). Several methods such as dye staining with methylene blue (MB) and tissue autofluorescence (AF) have been advocated to reduce the number of biopsies. We assessed their sensitivity and specificity compared with the standard approach - that is, endoscopy with $4 \mathrm{QB}$-in the surveillance of a mixed BO population.

Patients and methods: Thirty five consecutive BO patients (mean age 64.9 years; 30 men, five women) were included in the study. AF endoscopy was followed by high resolution video endoscopy (VE) plus tissue staining with $0.5 \% \mathrm{MB}$. Biopsies were taken from any suspicious area found on any of the above tests, in addition to $4 \mathrm{QB}$ every $2 \mathrm{~cm}$. The results were classified as either positive or negative for the various tests used. Histopathological results were used as the reference standard.

Results: In the 35 study patients, a total of 345 biopsies showed low grade dysplasia (LGD) in 88 biopsies, high grade dysplasia (HGD) in 19 biopsies, and carcinoma in 12 biopsies. The sensitivity and specificity rates for $\mathrm{AF}$ and $\mathrm{MB}$ for the diagnosis of cancer or dysplasia versus $\mathrm{BO}$ mucosa without dysplasia were $21 \% / 91 \%$ and $37 \% / 91 \%$, respectively. $4 Q B$ revealed five cancer/HGD areas and 76 LGD areas not detected by AF, MB, or VE. The additional yield of MB and AF over VE with $4 Q B$ concerned only one HGD area (in the vicinity of a cancer) and seven LGD areas.

Conclusions: Due to their low sensitivity, $A F$ and $M B$ are not suitable techniques for reducing the high numbers of routine biopsies needed for finding additional foci of HGD or cancer. Careful endoscopic observation and stepwise four quadrant biopsy therefore still represent the gold standard for surveillance of Barrett's oesophagus.
\end{abstract}

bee he incidence of distal oesophageal adenocarcinoma has been increasing recently, and a link with Barrett's oesophagus $(\mathrm{BO})$ has been established. In patients with $\mathrm{BO}$, the incidence of carcinoma ranges from 1 in 52 patient years ${ }^{1}$ to 1 in 208 patient years ${ }^{2}$ in a number of reports. ${ }^{13-6}$ As these tumours develop in a dysplasia-carcinoma sequence, surveillance of patients using endoscopy with stepwise biopsy is generally recommended. The gold standard for BO surveillance, known as the Seattle protocol, ${ }^{7}$ includes four quadrant biopsies (4QB) every $1-2 \mathrm{~cm}$ along the endoscopically visible length of BO. An approach of this type, requiring a large number of biopsies along the entire length of the Barrett's segment, is time consuming and may not be free of risk. It is also hampered by potential sampling errors as the majority of dysplastic areas are not visible on standard endoscopy. Efforts have therefore been made to enhance the visibility of suspicious areas in $\mathrm{BO}$ using various endoscopic techniques, ranging from methylene blue (MB) staining to technically more sophisticated methods such as tissue autofluorescence (AF). Only a few studies on MB staining have been conducted, ${ }^{8-14}$ and the results of AF studies have been published only in abstract form or in reviews. ${ }^{15-19}$

We therefore conducted a prospective study evaluating the sensitivity and specificity of $\mathrm{MB}$ staining and tissue $\mathrm{AF}$ measurement using high resolution video endoscopy (VE) with $4 \mathrm{QB}$ as the reference method in patients with established BO. The gold standard for the final diagnosis of normal BO or any form of dysplasia or cancer was histology of biopsies. Endoscopy with biopsy is the current practice in BO surveillance (biopsy of any endoscopically suspicious lesions plus $4 \mathrm{QB}$ ) with which any new method has to be compared.
Two questions were posed which we hoped would be answered by the results of our study:

(1) Can the number of random biopsies (4QB) be reduced so that only targeted biopsies guided by endoscopy in addition to $\mathrm{AF}$ and $\mathrm{MB}$ have to be taken, which would require excellent sensitivity and good specificity of MB and/or AF?

(2) Can the diagnostic accuracy of the standard approach (VE plus $4 \mathrm{QB}$ ) be increased by $\mathrm{MB}$ and/or AF? This would be the case if a significant number of correctly positive $\mathrm{MB}$ and/or $\mathrm{AF}$ areas were found which were macroscopically not visible and outside of the 4QB spots.

\section{PATIENTS AND METHODS \\ Patients}

It was initially planned that the study would include 50 patients, with an interim analysis after 35 patients. These numbers were chosen as it was expected that a minimum of 10 biopsies had to be taken from each patient to ensure reliable statistical results (with this number of patients 350 biopsies were investigated). Due to the poor results (see below) it was therefore decided to discontinue the study after the interim analysis. A total of 35 consecutive patients (30 men and five women; mean age 64.8 years; range 29-78) with histologically verified $\mathrm{BO}$ were therefore included in the

Abbreviations: $4 \mathrm{QB}$, four quadrant biopsies; $\mathrm{BO}$, Barrett's oesophagus; $\mathrm{NBO}, \mathrm{BO}$ without dysplasia; $\mathrm{MB}$, methylene blue; $\mathrm{AF}$, autofluorescence; $V E$, video endoscopy; LGD, low grade dysplasia; HGD, high grade dysplasia. 
analysis. Fifteen had a significant hiatal hernia $(>3 \mathrm{~cm}$ in diameter), five had a small hiatal hernia $(<3 \mathrm{~cm}$ in diameter), and 15 patients did not have a hiatal hernia. If inflammatory lesions (reflux oesophagitis, erosions) were found at endoscopy, the patient received at least two weeks' treatment with proton pump inhibitors before a repeat endoscopy for BO surveillance was carried out; this was negative for reflux oesophagitis in all cases. Twenty nine patients had long segment BO $(>3 \mathrm{~cm}$, assessed on endoscopy as the distance between the $\mathrm{Z}$ line and the proximal gastric folds) and six patients had short segment Barrett's ( $1-3 \mathrm{~cm}$ ).

The clinical indications for surveillance endoscopy in the study patients were as follows.

- Routine surveillance of patients with known BO without $(n=18)$ or with $(n=8)$ only low grade dysplasia (LGD).

- Focused surveillance to help reach a treatment decision in patients with $\mathrm{BO}$ and high grade dysplasia (HGD) $(\mathrm{n}=1)$.

- Patients with a diagnosis of cancer, for treatment planning-for example, mucosectomy versus photodynamic therapy versus surgery $(\mathrm{n}=8)$.

- Patients with a new diagnosis of BO undergoing surveillance for the first time $(n=18)$.

Each patient was included only once in the study, even if he or she was examined twice during the study period. After endoscopy, outpatients $(n=20)$ were monitored clinically for at least 4-5 hours, and were asked to report any adverse events occurring after discharge.

\section{Methods}

After patients had given informed consent to participation in the study, endoscopic examinations were conducted by two experienced examiners, with patients receiving intravenous sedation with midazolam and propofol. The relevant sequences of all diagnostic steps taken (see below) were documented on videotape. Endoscopic locations documented during the examination (using centimetre distances from the incisors) were the $\mathrm{Z}$ line, the lower oesophageal sphincter area (distal narrowing of the oesophagus, end of the proximal gastric folds), and the diaphragmatic hiatus (best visible with the patient breathing). The four quadrants were defined in relation to the gastric anatomy, 12 o'clock being equivalent to the lesser gastric curvature. Each lesion that was found to be positive using any of the methods described below was carefully documented during the examination with regard to its longitudinal and circumferential position to locate it on the final biopsy session; in doubtful cases, video documentation allowed review during the examination.

The first examination conducted in all patients was tissue $\mathrm{AF}$, with the Xillix/Olympus laser induced fluorescence endoscopy in the gastrointestinal tract (LIFE-GI) system, using a fibreglass endoscope. This technique is based on the principle that endogenous fluorophores (such as flavines, collagen, $\mathrm{NADH}$, and porphyrins) are excited by monochromatic blue laser light at a wavelength of $437 \mathrm{~nm}$. Depending on the characteristics of the tissue, light is reflected as green light (normal tissue) or dark red light (dysplastic areas), corresponding to a higher loss of energy in the reflected light. ${ }^{20}$ These spectra are detected using a red-green camera on the fibreglass endoscope and are converted by a dedicated software program into a visible real time image. Each lesion positive on AF was carefully documented concerning distance (in $\mathrm{cm}$ ) from the incisors and position at the circumference to locate it for later biopsy (see below).

After the AF examination, a standard endoscopic examination was carried out using a high resolution standard video endoscope (Olympus GIF-140). Any macroscopically suspicious areas (ulcers, depressed and elevated lesions, irregular areas, areas of distinct colour change) were again documented carefully with regard to their longitudinal and circumferential location. After this careful observation process, MB staining was carried out, as described previously. ${ }^{10}{ }^{12}$ Briefly, about two minutes after washing and spraying with $10 \%$ $\mathrm{N}$-acetylcysteine to remove residual mucus, $\mathrm{MB} 0.5 \%$ was applied circumferentially over the entire length of the Barrett's segment using a special spray catheter (Olympus PW-5L). After a further two minutes, rinsing with various volumes of water (100-200 ml) followed. Inhomogeneously stained areas or areas with weak staining were recorded as positive.

Finally, biopsies were taken only at the end of the endoscopic evaluations: firstly, from any areas regarded as suspicious using high resolution VE and, in addition, from areas positive on any of the two imaging tests (MB and AF), even if these areas were completely normal on VE; the precise location of $\mathrm{AF}$ and $\mathrm{MB}$ positive areas was documented previously. Secondly, in addition, 4QB at 12, 3, 6, and 9 o'clock were taken every $2 \mathrm{~cm}$ from the rest of $\mathrm{BO}$ which was normal on VE and negative on $\mathrm{MB}$ and $\mathrm{AF}$; if a suspicious area (that is, positive on $\mathrm{VE}, \mathrm{AF}$, or $\mathrm{MB}$ ) was located at one of the $4 \mathrm{QB}$ areas (that is, precisely at the same distance from the incisors and at either $12,3,6$, or 9 o'clock), no additional biopsy was taken from the same quadrant. Biopsies were taken from the presumed distal $\mathrm{BO}$ margin (proximal gastric folds) to the proximal end marked by the $\mathrm{Z}$ line. Biopsies were taken using an Olympus $7 \mathrm{~mm}$ standard spiked forceps (Olympus FB240Q-1) as it has been shown that the jumbo forceps is not superior to the conventional biopsy technique. ${ }^{21}$

\section{Histopathology}

Histology from (a) targeted biopsies of any macroscopically suspicious lesion and in addition (b) routine 4QB from macroscopically normal mucosa were used as the gold standard for the diagnosis of normal BO mucosa, LGD, HGD, and cancer. Biopsies were routinely fixed in formalin and paraffin embedded. Serial sections $(5 \mu \mathrm{m})$ were cut and stained with haematoxylin and eosin. Diagnoses were made according to the WHO classification. ${ }^{22}$ Briefly, BO was diagnosed if intestinal metaplasia with columnar and goblet cells were present. Epithelial atypia in $\mathrm{BO}$ was assessed as negative, indefinite, or positive for dysplasia (that is, intraepithelial neoplasia). If dysplasia was present, it was classified as low grade (synonymous with mild or moderate dysplasia) or high grade (synonymous with severe dysplasia and carcinoma in situ). All biopsies were seen by one experienced gastrointestinal histopathologist (MW).

\section{Statistical analysis}

For each method (AF, MB, VE), the sensitivity and specificity for detecting cancer/dysplasia were calculated, with histology being used as the reference standard. Accuracy rates of the respective methods were tested for statistically significant differences using the $\chi^{2}$ test and Fisher's exact test. Fisher's exact test was used if the numbers were too low for the $\chi^{2}$ test $(n<20)$. A p value below 0.05 was regarded as statistically significant.

In this study sensitivity was calculated separately for all three lesions (cancer, LGD, and HGD) as correct number of positives out of the total number of positive biopsies; specificity was calculated as correct number of negative biopsies among all negative biopsies. Positive and negative predictive values were calculated accordingly.

\section{RESULTS}

\section{Patient characteristics}

In the 35 patients included, the most advanced diagnoses per patient relative to the reference standard (4QB) were as follows: invasive carcinoma in eight patients, HGD alone in one patient, LGD alone in eight patients, and BO without dysplasia (NBO) in 18 patients. Carcinoma and dysplasia (HGD and LGD) frequently coexisted; details are shown in table 1 . In 
Table 1 Final diagnoses in the 35 study patients

\begin{tabular}{lrll}
\hline & $\mathrm{n}$ & $\begin{array}{l}\text { Additional } \\
\text { HGD }\end{array}$ & $\begin{array}{l}\text { Additional } \\
\text { LGD }\end{array}$ \\
\hline Patient related diagnoses* & & & \\
Normal BO without dysplasia & 18 & 0 & 0 \\
Carcinoma & 8 & 7 & 7 \\
HGD & 1 & - & 1 \\
LGD & 8 & - & - \\
Biopsy related diagnoses & & & \\
Normal biopsies & 226 & & \\
Biopsies with LGD & 88 & & \\
Biopsies with HGD & 19 & & \\
Biopsies with cancer & 12 & & \\
\hline
\end{tabular}

* Most advanced diagnosis based on histology of biopsies. BO, Barrett's oesophagus; HGD, high grade dysplasia; LGD, low grade dysplasia.

24 patients, the examination conducted for the study confirmed an earlier diagnosis (at other institutions) with regard to carcinoma, $\mathrm{HGD}$, LGD, or NBO; previous diagnoses were upgraded (for example, from NBO to LGD, or from dysplasia to carcinoma) in 11 patients.

A total of $168 \mathrm{~cm}$ of BO mucosa were screened and a total of 530 biopsies were taken, 345 of which $(65 \%)$ demonstrated Barrett's epithelium; the remaining biopsies yielded gastric/ cardiac epithelium, all from the distal BO area. As shown in table 1, 226 biopsies yielded normal BO, 88 showed LGD, 19 showed HGD, and 12 indicated cancer. No complications occurred during the study. The following results were based on two examiners, between whom there was no significant difference in accuracy $\left(\chi^{2}\right.$ test: $\left.p>0.05\right)$.

\section{Accuracy of MB and AF}

The results of $\mathrm{MB}$ and $\mathrm{AF}$ compared with the final histopathological diagnoses are shown in table 2 (biopsy related) and table 3 (patient related). Biopsy related sensitivity and specificity rates for $\mathrm{MB}$ and $\mathrm{AF}$ in diagnosing cancer or dysplasia versus normal Barrett's mucosa were $21 \% / 91 \%$ and $37 \% / 91 \%$, respectively, for the total of 345 biopsies (table 2). As a positive result on $\mathrm{AF}$ or $\mathrm{MB}$ could not differentiate between cancer, HGD, and LGD, positive predictive values were calculated for all three conditions: $49 \%$ for $\mathrm{AF}$, $58 \%$ for $\mathrm{MB}$, with a prevalence of 35\% (cancer, HGD, and LGD) for 345 biopsies. Negative predictive values were related to the 226 biopsies with normal BO epithelium: $69 \% \%$ for AF and $72 \%$ for MB.

With regard to patients, sensitivity and specificity rates were $71 \%$ and $50 \%$ for $\mathrm{MB}$ and $59 \%$ and $78 \%$ for $\mathrm{AF}$ (table 3 ).

\section{Accuracy of standard VE}

Compared with the histopathological gold standard, VE alone - without AF and/or MB-had a sensitivity of $43 \%$ and 95\% (biopsy related) and 79\% and 61\% (patient related). These values (see tables 2,3) indicate that the same or even slightly better accuracy was reached by standard VE alone compared with $\mathrm{MB}$ and $\mathrm{AF}$; positive and negative predictive values of VE were $73 \%$ and $72 \%$. However, $12 \%$ of cancerous and $58 \%$ of HGD areas were missed by endoscopy alone.

\section{Additional value of $A F$ and $M B$}

In a further detailed analysis, the additional value of biopsies directed by $\mathrm{AF}$ and $\mathrm{MB}$ staining was as follows:

- Cancers diagnosed by AF and/or MB staining, but not visible or suspected on VE, were not encountered in the study. However, four spots of $H G D$ were detected by MB which however were within the 4QB protocol; one HGD spot was identified by AF which was outside of the 4QB reach.

- Seven areas of $L G D$ (in four patients) were found by AF only (four outside of the 4QB protocol), and four areas of LGD (in two patients) by MB staining only (three outside of the $4 \mathrm{QB})$; these areas were all inconspicuous on VE.

Table 2 Results with autofluorescence (AF) and methylene blue (MB) staining compared with histological findings as the reference standard in 345 biopsies from patients with Barrett's osophagus (BO); the results of video endoscopy (VE) alone are also shown (with respect to the respective accuracy achieved, no statistically significant differences were found among the different methods used). A positive biopsy was from either a suspicious area on VE or any of the two methods tested (AF and $M B$ ), or from the additional four quadrant biopsies

\begin{tabular}{lclrrr}
\hline Cancer & 12 & True positive* & $3(25 \%)$ & $6(50 \%)$ & $8(67 \%)$ \\
\hline HGD & 19 & True positive* & $5(26 \%)$ & $9(47 \%)$ & $8(42 \%)$ \\
LGD & 88 & True positive* & $11(13 \%)$ & $12(14 \%)$ & $14(16 \%)$ \\
Normal BO & 226 & True negative† & $206(91 \%)$ & $205(91 \%)$ & $214(95 \%)$ \\
\hline
\end{tabular}

*Sensitivity of $A F, M B$, or VE in the detection of cancer, HGD, and $L G D$, respectively.

$\dagger$ Specificity of $A F, M B$, or $V E$ in the correct recognition of normal $B O$ (see text for positive and negative predictive values).

HGD, high grade dysplasia; LGD, low grade dysplasia.

Table 3 Results with autofluorescence (AF) and methylene blue (MB) staining compared with histological findings as the reference standard in the 35 patients with Barrett's osophagus (BO); the results of video endoscopy (VE) alone are also shown (with respect to the respective accuracy achieved, no statistically significant differences were found among the different methods used)

\begin{tabular}{lrllll}
\hline $\begin{array}{l}\text { Histolopathological } \\
\text { diagnosis* per patient }\end{array}$ & $\mathrm{n}$ & Method & AF & MB & VE alone \\
\hline Cancer & 8 & True positive & $3(38 \%)$ & $6(75 \%)$ & $7(88 \%)$ \\
HGD & 1 & True positive & $1(100 \%)$ & $1(100 \%)$ & $1(100 \%)$ \\
LGD & 8 & True positive & $3(38 \%)$ & $3(38 \%)$ & $4(50 \%)$ \\
Normal BO & 18 & Truenegative & $14(78 \%)$ & $9(50 \%)$ & $11(61 \%)$ \\
\hline
\end{tabular}

* Most advanced diagnoses per patient-that is, a patient with 10 normal biopsies, four LGD, and one HGD is a "HGD patient".

HGD, high grade dysplasia; LGD, low grade dysplasia. 


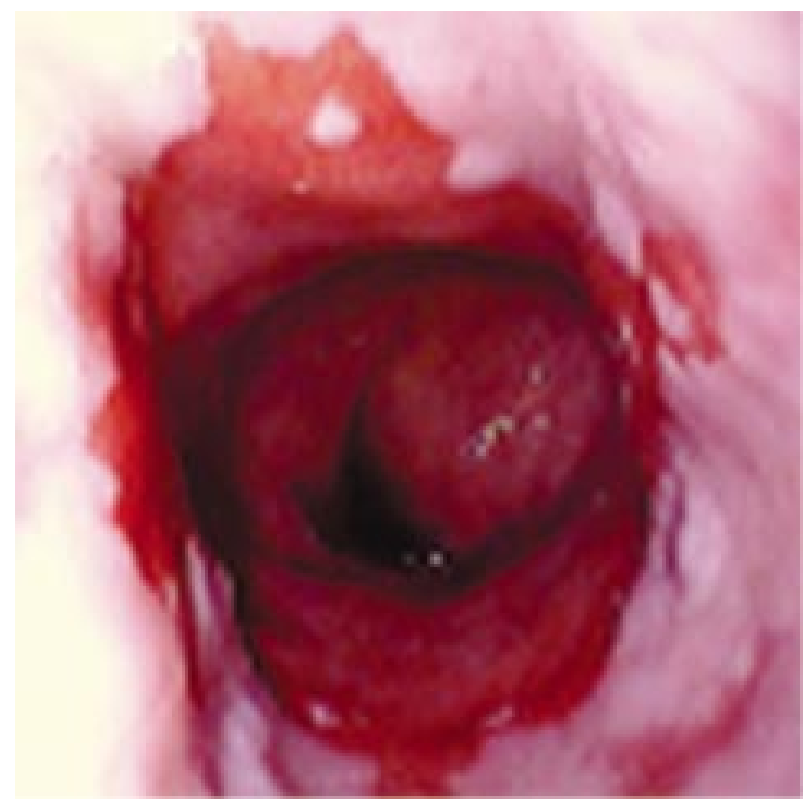

Figure 1 Video endoscopy. Example of a patient with Barrett's oesophagus without dysplasia.

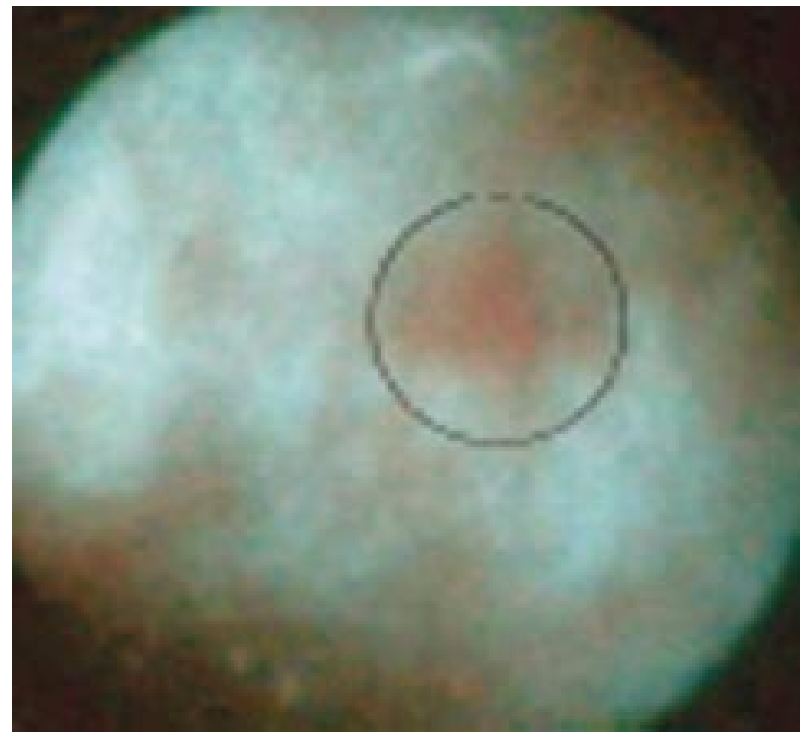

Figure 2 Example of a patient with Barrett's oesophagus with high grade dysplasia, negative on video endoscopy but positive on autofluorescence.

- In summary, compared with the standard approach with VE plus 4QB, only one additional HGD area and seven LGD areas would have been detected by $\mathrm{AF}$ and $\mathrm{MB}$ as these areas were endoscopically not visible by standard VE and were located outside the 4QB protocol. On the other hand, the one HGD area and three of the seven LGD areas were close to macroscopically visible cancers.

- Considering VE plus MB and AF together and comparing targeted biopsies using these three methods with the 4QB results, 76 LGD areas, four HGD areas, and one cancer would have been missed as they were only evident on the 4QB and were not visible by VE, AF, or MB. Examples are shown in figs $1-3$.

\section{DISCUSSION}

Surveillance examinations in patients with Barrett's oesophagus, a condition which carries a risk for the development of



Figure 3 Video endoscopy. Example of a patient with Barrett's oesophagus with low grade dysplasia, negative on video endoscopy but positive on methylene blue staining.

distal oesophageal adenocarcinoma, are time consuming and potentially risky, requiring acquisition of multiple biopsies around the luminal circumference every $2 \mathrm{~cm}$, and consequently an often lengthy period of sedation. ${ }^{7}$ Before 4QB, all endoscopically suspicious areas (showing elevation, depression, or colour changes) also have to be biopsied. Using this policy, sampling errors cannot be excluded as dysplastic changes per se are usually not visible even on high quality VE. Some studies comparing biopsy protocols with histopathological evaluation of resection specimens have shown that in $41-66 \%$ of patients with confirmed HGD, the lesions were missed prior to surgery even when stepwise endoscopic biopsy was used. ${ }^{23-25}$ However, most of these studies were carried out in patients undergoing resection for cancer and/or HGD; the situation may therefore be less worrisome in patients with $\mathrm{BO}$ with or without LGD due to the much lower prevalence of advanced lesions in these conditions.

For these reasons, targeted biopsy of lesions, invisible on standard endoscopy but becoming visible after staining or inducing fluorescence, is desirable to reduce the number of biopsies. The results of our study demonstrated that $\mathrm{MB}$ staining and tissue AF did not have high enough accuracy to replace systematic $4 \mathrm{QB}$. To attain this goal, sensitivity would have to be excellent (for example $>90 \%$ ) to ensure that no or only a very few areas of invisible cancer or dysplasia would be missed using MB or AF targeting alone for obtaining biopsies. A somewhat lower specificty (for example, approximately $80 \%$ ) could be accepted as this would mean superfluous biopsies in some instances. VE alone showed a similar (but also insufficient) accuracy in detecting dysplastic and cancerous areas; however, approximately $10 \%$ of cancerous and nearly $60 \%$ of HGD areas were missed by VE. On the other hand, one could argue that 4QB still has to be done but staining or fluorescence could highlight some areas not recognised by standard VE and not covered by the standard 4QB which are histologically positive for dysplasia or cancer. Unfortunately, both methods tested in our study again failed to reach this goal. 
Several endoscopic techniques, some of which are simple while others are more sophisticated, are currently being evaluated in the surveillance of BO. The easiest method is probably dye staining using MB. Staining involves cleaning the mucosal surface and spraying and washing off the dye after a few minutes. Evidence in the literature on the value of dye spraying in $\mathrm{BO}$ at present rests on a few original articles $^{8-11}$ and a series of abstracts. ${ }^{12-14}$ Unfortunately, these studies yielded conflicting results. Canto et al showed that using MB staining, 15\% more biopsies showing dysplasia and $31.5 \%$ more showing carcinoma were obtained, despite a lower overall number of endoscopic biopsies in comparison with standard 4QB. ${ }^{8}$ Similarly, Gossner et al found the same rate of dysplasia in endoscopic biopsies with $\mathrm{MB}$ staining directed biopsy as with $4 \mathrm{QB}$ but with significantly fewer biopsies being needed..$^{13}$ In contrast, other authors have not observed a higher rate of dysplasia in MB directed surveillance biopsies than in conventional ones. ${ }^{12}$ Gangarosa and colleagues ${ }^{10}$ found that only $12.5 \%$ of unstained mucosal areas had histologically confirmed (low grade) dysplasia. In our study, we did not reproduce the good results of MB directed biopsy. In total, a very low number of MB directed biopsies yielded additional dysplasia/carcinoma, and the sensitivity and specificity rates did not reach the standards necessary to obviate the need for routine $4 \mathrm{QB}$. During the study-despite carefully adhering to the MB staining procedure as described in the literature ${ }^{8}{ }^{11}$ we found that the MB staining method was subjective and dependent on the intensity of washing after staining, as well as on other factors.

A similarly poor level of sensitivity was found with tissue AF using the LIFE-GI system. The available literature on this topic does not include any full publications; results have been presented in abstract form ${ }^{15-17}$ and in review articles. ${ }^{19} 20$ Haringsma and Tytgat ${ }^{20}$ used AF for the detection of HGD and early cancer, with sensitivity and specificity rates of $87 \%$ each. However, standard endoscopy produced similar results (76\% sensitivity and $93 \%$ specificity), and all of the lesions that were positive on AF were detectable by standard endoscopy. Performing a high resolution endoscopy examination before applying AF may therefore have introduced some interpretation bias. LGD on the other hand was detected with a sensitivity of only $18 \%$, a rate similar to ours. Du Vall et al examined only patients with BO associated cancer. They also reproduced all macroscopically visible lesions on AF but found only one additional area with HGD out of a total of 43 cases. The sensitivity and specificity rates of conventional VE were not stated. ${ }^{15}$

There may be several reasons for the divergent results with endoscopy associated techniques in the detection of dysplasia in BO. Methodological factors, such as the extent to which examiners are blinded, play a role. In the present study, we evaluated AF (using fibreglass endoscopes) before high quality VE was performed. This may have reduced the bias of examiners towards rating areas that had previously been identified as positive on VE as also being positive on AF. Another fact is that most studies included a large number of patients with HGD and cancer in whom lesions were already known and patients had specifically been referred for treatment planning. In the present study, the majority of patients were presenting for routine surveillance of $\mathrm{BO}$ (two thirds of the study population).

In summary, the results of our study allow us to answer the following questions.

(1) How accurate are AF and MB? Comparison of AF and MB with the standard approach consisting of VE with targeted plus 4Q biopsies showed that both $\mathrm{AF}$ and $\mathrm{MB}$ were significantly inferior to the standard approach.

(2) Can AF and/or MB increase the accuracy of the standard approach consisting of VE and targeted plus 4QB? Only one HGD and seven LGD areas were found outside the standard approach but mostly in the vicinity of gross cancers.
(3) Can AF and/or MB supplement VE so that only targeted biopsies are necessary and 4QB can be made superfluous? Comparison of VE plus AF and MB with targeted biopsies by these three methods on the one hand and additional 4QB biopsy on the other hand showed that all three endoscopic methods failed to reveal five cancers/HGD and almost all LGD areas which were obvious only from 4QB. Although the clinical significance of LGD is debated, ${ }^{26-28}$ the five other cases demonstrate that 4QB cannot be replaced.

In recent years, several technically sophisticated techniques for detecting invisible abnormalities have been presented ${ }^{29-39}$ such as tissue spectroscopy, optical coherence tomography, and tissue fluorescence techniques. In principle, the LIFE-GI technique offers the advantage of real time imaging of the entire oesophageal circumference. However, this advantage is offset by the relatively low natural fluorescence of tissue. It is conceivable that modifications of the technique using a photosensitiser might enhance the diagnostic accuracy (photodynamic diagnosis $)^{29-32}$ but this has yet to be proved in larger trials. Preliminary results have shown that after administration of the sensitiser 5-aminolevulinic acid, dysplasia was detected in both cases. ${ }^{31}$ Spectroscopic techniques ${ }^{33}$ only allow small areas to be viewed at one time, and this may make screening of a long BO segment quite time consuming. In most studies published to date, only macroscopically visible areas have therefore been examined, and a search for invisible dysplasia in endoscopically normal mucosa has not been carried out, ${ }^{34}$ although from a clinical standpoint this is the facility that would be most needed. Svanberg et al evaluated a combination of endogenous and exogenous fluorescence (using photofrin). By calculating a quotient of exogenous and endogenous fluorescence, carcinomas and HGD were differentiated from normal Barrett's mucosa in 12 cases. ${ }^{35}$ With optical coherence tomography-another potentially useful technique - rather poor preliminary data have been published for detecting dysplasia in $\mathrm{BO}^{36} 37$

In conclusion, this prospective study in a mixed population of patients with $\mathrm{BO}$ found that $\mathrm{MB}$ staining and determination of tissue AF were not capable of increasing the diagnostic accuracy or replacing standard four quadrant biopsies. Future studies should therefore evaluate other techniques, and should also take methodological issues and practicability into account. At present however the stepwise four quadrant biopsy protocol currently in use must still be regarded as the reference method. Future directions for research might be either to narrow the biopsy intervals from $2 \mathrm{~cm}$ to $1 \mathrm{~cm}^{38}$ or to further develop and evaluate new techniques for detecting dysplastic areas that are not visible on standard endoscopy.

\section{Authors' affiliations}

K Egger, A Meining, R Ott, H-D Allescher, M Classen, T Rösch, Department of Internal Medicine II, Technical University of Munich, Munich, Germany

M Werner, H Höfler, Department of Pathology, Technical University of Munich, Munich, Germany

\section{REFERENCES}

1 Hameeteman W, Tytgat GNJ, Houthoff HJ, et al. Barrett's esophagus: development of dysplasia and adenocarcinoma. Gastroenterology 1989;96:1249-56

2 Drewitz DJ, Sampliner RE, Garewal HS. Long term follow-up of Barrett's cohort. Am J Gastroenterol 1997:92:212-15.

3 Dent J, Bremner CD, Collen M, et al. Working party report to the World Congress of Gastroenterology, Sydney, 1990: Barrett's esophagus. J Gastroenterol Hepatol 1991;6:1-22.

4 Cameron AJ, Ott BJ, Payne WS. The incidence of adenocarcinoma in columnar-lined (Barrett's) esophagus. N Engl J Med 1985:313:857-9.

5 Spechler SJ, Robbins AH, Rubins HB, et al. Adenocarcinoma and Barrett's esophagus: an overrated risk? Gastroenterology 1984;87:927-33.

6 Spechler SJ. Endoscopic surveillance of patients with Barrett's esophagus: does the cancer risk justify the practice? Ann Intern Med 1987; 106:902-4. 
7 Levine DS. Management of dysplasia in the columnar-lined esophagus. Gastroenterol Clin North Am 1997;26:613-34.

8 Canto MI, Setrakian S, Willis J, et al. Methylene blue-directed biopsies improve detection of intestinal metaplasia and dysplasia in Barrett's esophagus. Gastrointest Endosc 2000:51:560-8.

9 Canto MI, Setrakian S, Petras RE, et al. Methylene blue selectively stains intestinal metaplasia in Barrett's esophagus. Gastrointest Endosc 1996;44: 1-7

10 Gangarosa LM, Halter S, Mertz H. Methylene blue staining and endoscopic ultrasound evaluation of Barrett's esophagus with low grade dysplasia. Dig Dis Sci 2000;45:225-9.

11 Canto MI. Vital staining and Barrett's esophagus. Gastrointest Endosc 1999;4:12-16

12 Ray MB, Mayfield-Stokes S, Cecil B, et al. Results of methylene blue-directed biopsy are similar to conventional biopsy for the diagnosis of intestinal metaplasia and dysplasia in Barrett's esophagus. Gastrointest Endosc 1997;47:AB28

13 Gossner L, Pech O, May A, et al. Methylene blue staining for the detection of dysplasia or mucosal cancer in Barrett's esophagus: prospective trial. Gastroenterology 1999:110:A414.

14 Gossner L, May A, Pech O, et al. Chromoendoskopie mit Methylenblau bei Barrettösophagus mit schweren Dysplasien oder Frühkarzinom. Z Gastroenterol 2000;38:663

15 Du Vall GA, Saidi R, Kost J, et al. Real-time light-induced fluorescence endoscopy (LIFE) in the gastrointestinal (GI) tract. Gastrointest Endosc 1997;45:AB28.

16 Haringsma J, Leewen M, Tytgat GNJ. Endoscopic localization of dysplasia in laser-induced fluorescence. Gastroenterology 1997;112:A576.

17 Haringsma J, Prawirordirdjo W, Tytgat GNJ. Accuracy of fluorescence imaging of dysplasia in Barrett's esophagus. Gastroenterology 1999;116:A418.

18 Stepp H, Sroka R, Baumgartner R. Fluorescence endoscopy of gastrointestinal diseases: basic principles, techniques, and clinical experience. Endoscopy 1998:30:379-86.

19 Marcon NE, Wilson BC. The value of fluorescence techniques in gastrointestinal endoscopy-better than the endoscopist's eye? Part 2: the North American experience. Endoscopy 1998;30:419-21.

20 Haringsma J, Tytgat GN. The value of fluorescence techniques in gastrointestinal endoscopy-better than the endoscopist's eye? Part 1: the European experience. Endoscopy 1998;30:416-18.

21 Falk G, Rice T, Goldblum J, et al. Jumbo biopsy protocol still misses unsuspected cancer in Barrett's esophagus with high-grade dysplasia. Gastroenterology 1999:49:170-6.

22 Werner M, Flejou JF, Haunaut $P$, et al. Adenocarcinoma of the esophagus. In: Aaltonen LA, Hamilton S, Kleihues P, et al, eds. WHO classification of tumors: pathology and genetics of tumors of the digestive system. Berlin: Springer, 2000:16-22.

23 Edwards $M J$, Gable DR, Lentsch $A B$, et al. The rationale for esophagectomy as the optimal therapy for Barrett's esophagus with high-grade dysplasia. Ann Surg 1996;223:585-9.
24 Heitmiller RF, Redmond M, Hamilton SR. Barrett's esophagus with high-grade dysplasia. Ann Surg 1996;224:66-71.

25 Peters JH, Clark GW, Ireland AP, et al. Outcome of adenocarcinoma arising in Barrett's esophagus in endoscopically surveyed and nonsurveyed patients. J Thorac Cardiovasc Surg 1994;108:813-21.

26 Sharma P, Weston AP, Morales T, et al. Relative risk of dysplasia for patients with intestinal metaplasia in the distal oesophagus and in the gastric cardia. Gut 2000;46:9-13.

27 Montgomery E, Goldblum JR, Greenson JK, et al. Dysplasia as a predictive marker for invasive carcinoma in esophagus: a follow-up study based on 138 cases from a diagnostic variability study. Hum Pathol 2001;32:379-88

28 Weston AP, Sharma P, Topalovski M, et al. Low-grade dysplasia in Barrett's esophagus: variable fate during long-term follow-up. Gastroenterology 1999;116:AB G1540.

29 Messmann H. Fluoreszenzendoskopie in der Gastroenterologie. Z Gastroenterol 2000;38:21-30.

30 Messmann H, Knuchel R, Endlicher E, et al. Photodynamic diagnosis of gastrointestinal precancerous lesions after sensitization with 5-aminolevulinic acid: a pilot study (in German). Dtsch Med Wochenschr 1998; 123:515-21.

31 Messmann H, Knuchel R, Baumler W, et al. Endoscopic fluorescence detection of dysplasia in patients with Barrett's esophagus, ulcerative colitis, or adenomatous polyps after 5-aminolevulinic acid-induced protoporphyrin IX sensitization. Gastrointest Endosc 1999;49:97-101.

32 von Holstein CS, Nilson AM, Andersson-Engels S, et al. Detection of adenocarcinoma in Barrett's esophagus by means of laser-induced fluorescence. Gut 1996;39:711-16.

33 Panjehpour M, Overholt BF, Vo-Dinh T, et al. Endoscopic fluorescence detection of high grade-dysplasia in Barrett's esophagus. Gastroenterology 1996;111:93-101

34 Wallace MB, Perelman LT, Backman V, et al. Endoscopic detection of dysplasia in patients with Barrett's esophagus using light-scattering spectroscopy. Gastroenterology 2000;119:677-82.

35 Svanberg K, Klintberg C, Nilsson A, et al. Laser-based spectroscopic methods in tissue characterization. Ann N Y Acad Sci 1998; 838: 123-9.

36 Brand S, Bonero JM, Bouma BE, et al. Optical coherence tomography in the gastrointestinal tract. Endoscopy 2000;32:796-803.

37 Jäckle S, Gladkova N, Feldchtein F, et al. In vivo endoscopic optical coherence tomography of esophagitis, Barrett's esophagus, and adenocarcinoma of the esophagus. Endoscopy 2000;32:750-5.

38 Reid BJ, Blount PL, Feng Z, et al. Optimizing endoscopic biopsy detection of early cancers in Barrett's high-grade dysplasia. Am J Gastroenterol 2000;95:3089-96.

39 Andersson-Engels S, af Klinteberg C, Svanberg K, et al. In vivo fluorescence imaging for tissue diagnosis. Phys Med Biol 1997;42 815-24. 